\title{
Major vascular complications in Behçet's syndrome
}

\author{
B. Anthony ENoch \\ B.Sc., M.B., Ch.B.(Liverpool) \\ Medical Registrar \\ T. C. L. KHOO \\ M.R.C.S.(England), L.R.C.P.(London) \\ Medical Registrar
}

\author{
J. L. Castillo-Olivares \\ L.M.C., D.M.(Madrid) \\ Research Fellow \\ R. G. GRAINGER \\ M.D.(Leeds), M.R.C.P.(London), D.M.R.D., F.F.R. \\ Consultant Radiologist
}

\author{
L. HENRY \\ M.D.(Birmingham), M.R.C.P.(London), M.C.Path. \\ Senior Lecturer in Pathology \\ The Royal Hospital, Sheffield and Regional Cardio-Thoracic Unit, \\ Northern General Hospital, Sheffield
}

\begin{abstract}
Summary
Two patients with Behçet's syndrome are described. One patient developed spontaneous bilateral popliteal artery aneurysms and the other spontaneous thrombosis of the superior vena cava. These cases emphasize that primary inflammatory lesions of large vessels, with arterial aneurysm formation and thrombosis may be a feature of the syndrome.
\end{abstract}

\section{Introduction}

Since Behçet in 1937 described a triad of orogenital ulceration and ocular lesions, a number of additional features has been recognized as part of the complex now known as Behcet's syndrome. These include erythema nodosum, pustular dermatitis, arthritis, a variety of central nervous system lesions, abnormal skin sensitivity, and recurrent thrombophlebitis (Mounsey, 1966).

Involvement of large veins, possibly with thrombosis of the superior or inferior vena cava is a recognized though rare complication of the disease (Forman, 1960, 1967). Only five cases in which there were lesions affecting major arteries have so far been recorded in the literature (Mishima, Ishikawa \& Kawase, 1961; Oshima et al., 1963 (two cases) ; British Medical Journal, 1965; Hills, 1967). In this paper we present two cases of Behçet's syndrome, the first of which was complicated by the spontaneous development of bilateral popliteal artery aneurysms, and the second by spontaneous thrombosis of the superior vena cava.

\section{Case 1}

The patient, J.F., is a 16-year-old apprentice tool-maker. In September 1965, at the age of 15 years, he was referred to an ophthalmologist on account of irido-cyclitis. Subsequently he developed aphthous ulceration of the oral mucosa and erythema nodosum. In spite of the absence of ano-genital lesions, a tentative diagnosis of Behçet's disease was made. The ocular manifestations were treated with topical steroids and mydriatics, and in June 1966 oral steroids were commenced.

On 3 November 1966 he was referred to the Professorial Medical Unit of the Sheffield Royal Hospital. Within the preceding 3 weeks there had been marked deterioration in vision, general malaise with anorexia, fever, and recurrent arthralgia in both legs.

On examination, he was pale and febrile $\left(100^{\circ} \mathrm{F}\right)$. He was of small stature with unbroken voice, absent facial and axillary hair and scanty pubic hair; the testes were small. A few red macules were present on the left upper arm. There was swelling and restricted movement of the right ankle joint and superficial thrombophlebitis of the right calf. The eyes showed severe bilateral pan-uveitis with marked impairment of visual acuity (R $3 / 60 \mathrm{~L} 1 / 36)$. The anogenital region was normal. Pustules developed at sites of venepuncture. He improved dramatically following treatment with ACTH and oxyphenbutazone. The oral lesions responded to hydrocortisone pellets and the uveitis was controlled by topical atropine and hydrocorti- 
sone. Anaemia, which was associated with iron and folic acid deficiency, and for which no cause was found, responded to appropriate oral supplements. The patient was discharged on maintenance therapy with ACTH.

On 23 January 1967 he was re-admitted as an emergency because of severe pain and swelling of the left knee and coldness of the left foot, which had been present for 3 days. On examination, the left knee was held in $30^{\circ}$ of flexion, and the joint appeared swollen and tender. Flexion from $30^{\circ}$ was possible, but attempts to extend the joint beyond this angle caused severe pain behind the knee. In the left popliteal fossa there was a firm, ill-defined, pulsatile, tender swelling approximately $2.5 \mathrm{~cm}$ diameter over which a systolic bruit was audible. The left foot was cold and white. The dorsalis pedis pulse was present although much diminished, and the posterior tibial pulse was absent. The patient was afebrile and the heart was clinically normal apart from sinus tachycardia. The clinical diagnosis of a left popliteal artery aneurysm was confirmed on angiography by retrograde catheterization via the right femoral artery (Fig. 1a).

An aneurysm $(3.0 \times 4.0 \mathrm{~cm})$ was demonstrated on the left popliteal artery, situated behind the knee joint. The aneurysm retained the

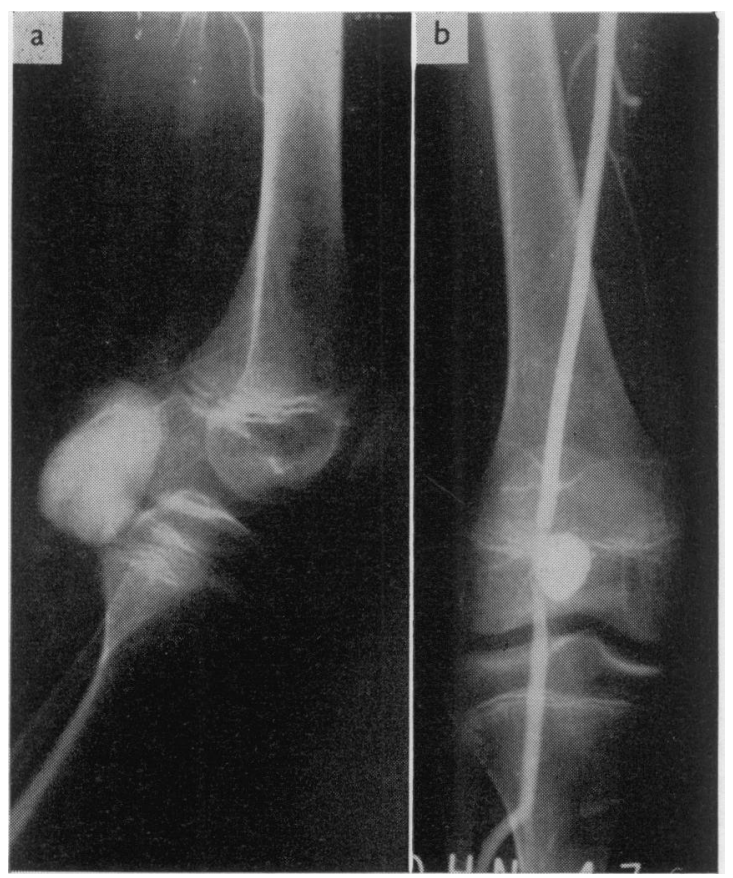

FIG. 1. (a) Left femoral arteriogram showing an aneurysm of the left popliteal artery (26 Jan). (b) Right popliteal artery aneurysm (14 Feb). contrast medium for several seconds and there was only faint opacification of the popliteal artery distal to it. The lower abdominal aorta and both iliac arteries appeared normal but the right popliteal artery was not examined.

Other investigations were as follows: Chest $\mathrm{X}$-ray: Essentially normal. Skeletal survey: No bone lesions seen. Haemoglobin: $13 \cdot 7 \mathrm{~g} / 100 \mathrm{ml}$. WBC: $12,000 / \mathrm{mm}^{3}$ (82\% neutrophils). ESR : $37 \mathrm{~mm} / \mathrm{hr}$ (Wintrobe). Platelets : $190,000 / \mathrm{mm}^{3}$. Bleeding time: $3 \frac{1}{2} \mathrm{~min}$. Plasma proteins: albumin $4 \cdot 3$, globulin $2 \cdot 5$, fibrinogen $0.34 \mathrm{~g} / 100 \mathrm{ml}$. Electrophoresis showed raised $\alpha_{2}-, \beta-$ and $\gamma-$ globulins. Blood cultures $(\times 3)$ : sterile after 7 days incubation. Whole blood clot lysis time: over $24 \mathrm{hr}$. Prothrombin time: $12 \mathrm{sec}$. Tanned red cell haemagglutination titre against oral mucosa $1: 320$ (controls approx. 1:40). LE cell preparation: no LE cells seen. Mantoux test: negative 1 in 1000 . Kveim test: negative. Toxoplasma antibody titre (dye test) 1:8. Karyotype: sex chromatin (buccal smear) negative, chromosome analysis (blood culture), 46 chromosomes. Sex chromosomes XY. Throat swab: no pathogens cultured. ASO titre: 400 Todd units. Serum calcium: $8 \cdot 1 \mathrm{mg} / 100 \mathrm{ml}$. WR : negative.

After blood cultures had been taken, penicillin and streptomycin were given as it was thought that the aneurysm might be mycotic. ACTH and oxyphenbutazone were given daily.

On 31 January the left popliteal fossa was explored (Mr D. G. Taylor). It was found that the true aneurysm had ruptured and much of the swelling was due to an extravasation of haematoma into the arterial wall causing a false aneurysm. The process involved the origin of the posterior tibial artery. The aneurysm was excised and replaced by an autogenous saphenous vein graft from the proximal popliteal artery to the anterior tibial artery. Histological studies of this aneurysm were not made. Postoperative progress was satisfactory.

During convalescence, scrotal ulcers typical of Behçet's syndrome were observed for the first time. It was noted that, although the patient had no symptoms referable to the right leg, the right popliteal pulse was unduly prominent and an arterial aneurysm at this site was suspected. On 14 February an aortogram was performed by retrograde catheterization via the right femoral artery. An aneurysm $(2.0 \mathrm{~cm}$ diameter) was demonstrated on the right popliteal artery. projecting medially in the femoral intercondylar notch (Fig. 1b). It had a very narrow neck and attachment from the popliteal artery. There was no interruption or delay in the passage of 
contrast medium distally beyond the aneurysm into the tibial arteries (Fig. 2).

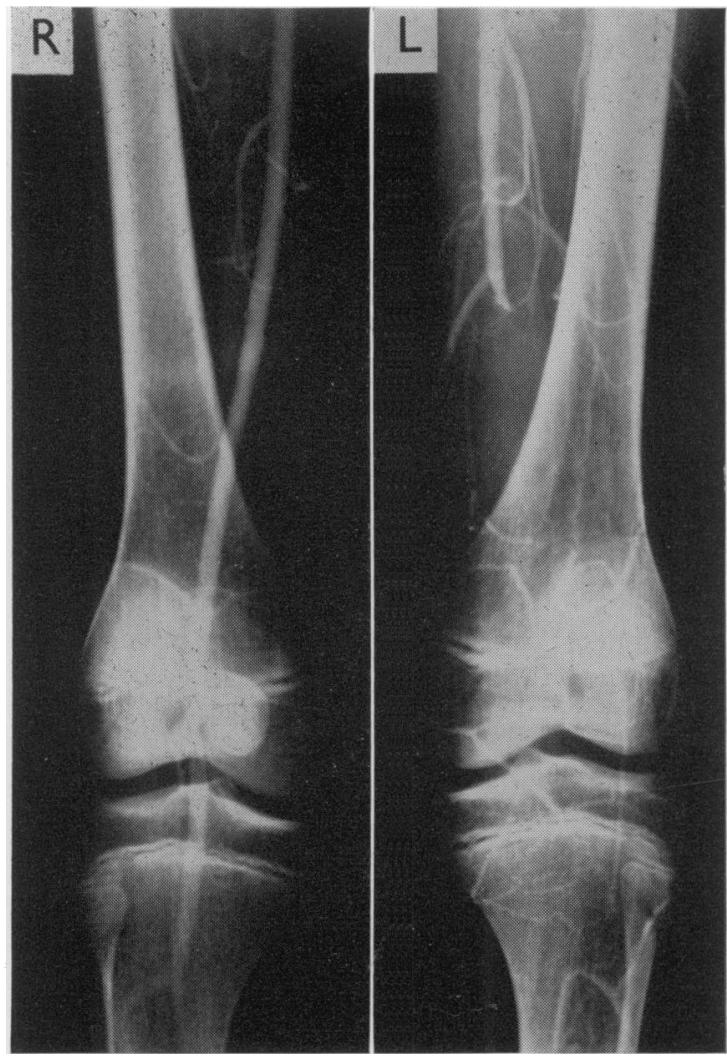

Fig. 2. Aortogram showing an aneurysm of the right popliteal artery and obstruction of the proximal left popliteal artery (14 Feb).

On the left side, there was complete obstruction at the upper end of the popliteal artery which showed no filling, presumably due to thrombosis. There was, however, an adequate collateral circulation providing good opacification of the tibial arteries. The aorta from the arch to the bifurcation, both iliac and both femoral arteries appeared normal.

On 5 May the patient developed pain and further swelling at the back of the right knee and on 9 May the right popliteal fossa was explored (Mr D. G. Taylor). A true saccular aneurysm (approx. $4.0 \times 3.0 \mathrm{~cm}$ ) containing thrombus was found on the distal portion of the popliteal artery. A roof-type autogenous saphenous vein graft was inserted, and most of the aneurysmal wall excised along with a macroscopically normal portion of popliteal artery immediately adjacent to the neck of the aneurysm. Histological studies (Fig. 3) of the

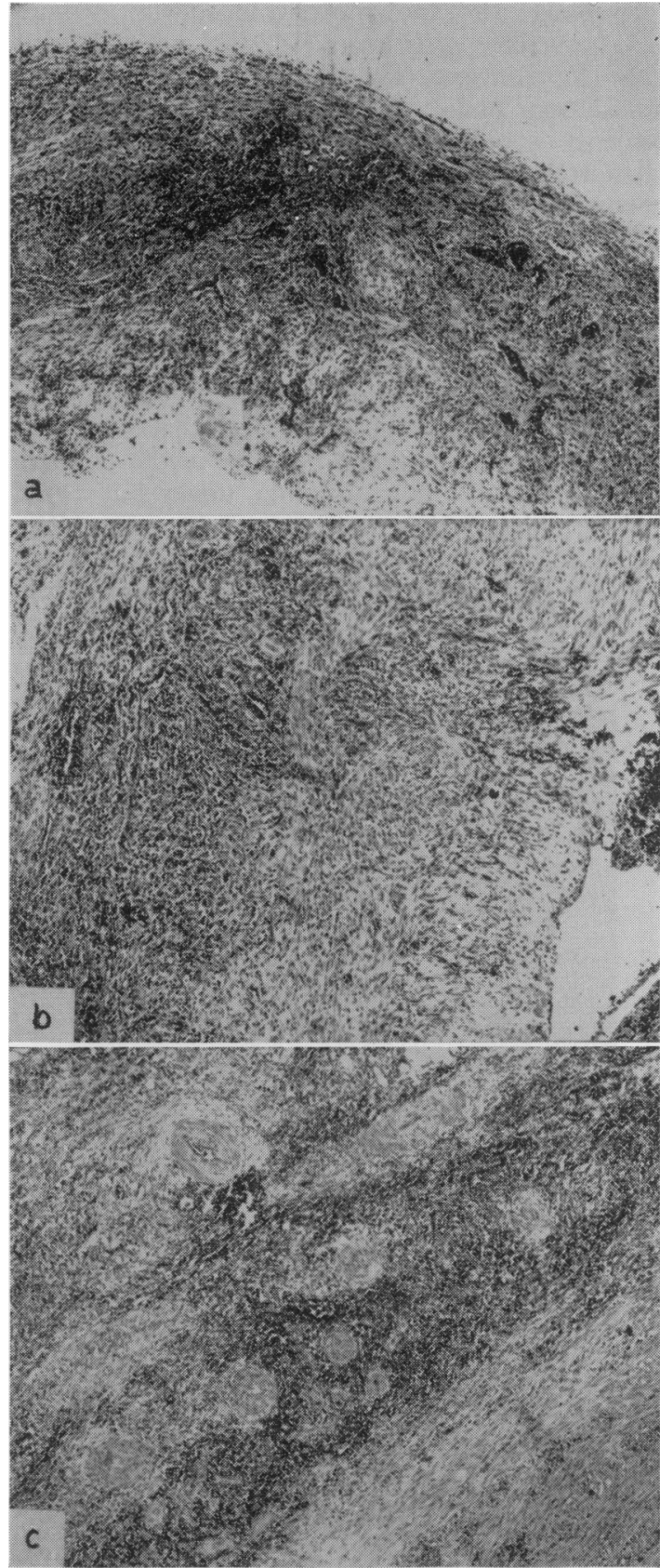

FIG. 3. (a) The wall of the aneurysm showing inflammation and destruction of the arterial wall. (H \& E, $\times 35$ ). (b) The wall of the aneurysm showing inflammation and formation of new fibrous tissue. (H \& E, $\times 42)$. (c) Adventitia adjacent to the aneurysm. The small blood vessels show obliterative endarteritis. $(H \& E, \times 42)$.

aneurysm showed a severe active inflammation with an exudate of plasma cells, lymphocytes, 
eosinophils and polymorph leucocytes. There was complete destruction of the arterial wall with replacement fibrosis and formation of granulation tissue. No muscle or elastic tissue elements could be identified. Areas of recent haemorrhage were present with haemosiderin in macrophages. The adventitial blood vessels and vasa vasorum showed an obliterative endarteritis but there was no fibrinoid necrosis. There was no granuloma or giant-cell formation and no foreign material, bacteria or fungi could be demonstrated. The lumen of the aneurysm contained recent laminated thrombus with early organization. The popliteal artery adjacent to the aneurysm showed similar but less severe changes with formation of granulation tissue but again no normal arterial wall could be seen. The popliteal vein was normal. A small lymph node near the aneurysm showed only reactive hyperplasia.

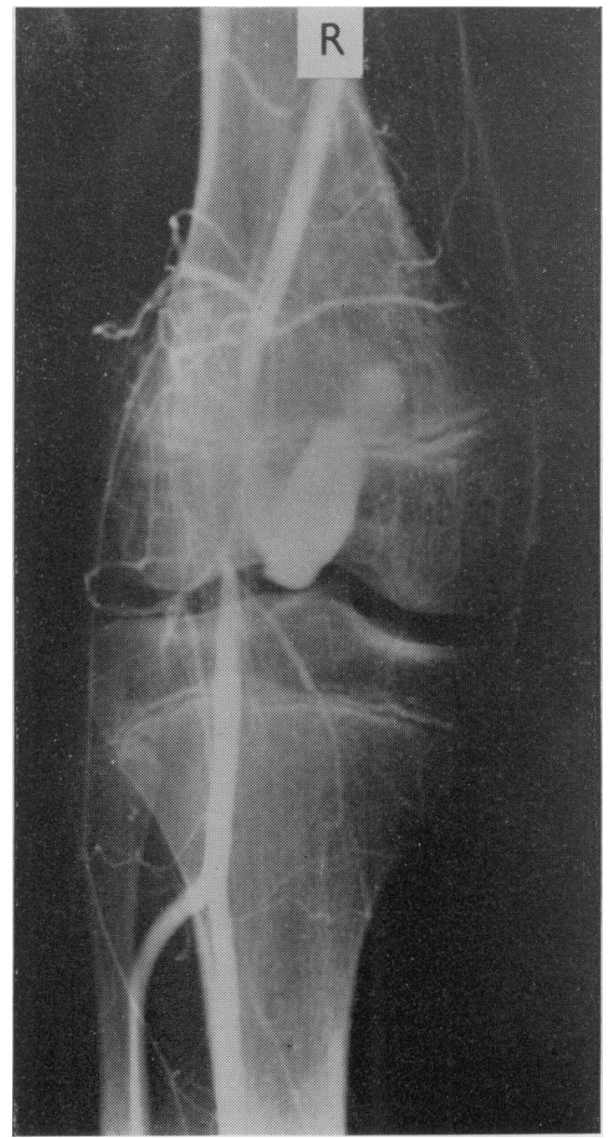

FIG. 4. Right femoral arteriogram showing recurrent right popliteal aneurysm (19 June).
Post-operatively, oral phenindione was given, but this was withdrawn at the end of 4 weeks on account of haematuria and bruising.

On 19 June the patient developed a recurrent painful swelling at the back of the right knee. A right femoral arteriogram demonstrated an irregular elongated aneurysm $(5.0 \times 2.0 \mathrm{~cm})$ projecting from the medial aspect of the right popliteal artery. This was at the same site as the aneurysm previously demonstrated (Fig. 4).

Three days later the right popliteal fossa was again explored and ligation of the popliteal artery was carried out proximal to the aneurysm which was left in situ. After operation the right foot was warm and a dorsalis pedis pulse was palpable, the vessel presumably being fed from collaterals. The patient made an uneventful recovery and was discharged home on a small maintenance dose of prednisolone.

\section{Case 2}

An obese 38-year-old builder, P.T., was admitted to the Northern General Hospital, Sheffield, on 25 November 1966, with increasing swelling of the neck of 1 week's duration. He gave a history of recurrent mouth ulceration for 3 years and episodic arthralgia for 4 years. Six weeks prior to admission he developed deep vein thrombosis of his right leg and at this time noticed scrotal ulceration.

On examination he had classical signs of superior mediastinal obstruction. Three punchedout ulcers were present on the scrotum and a

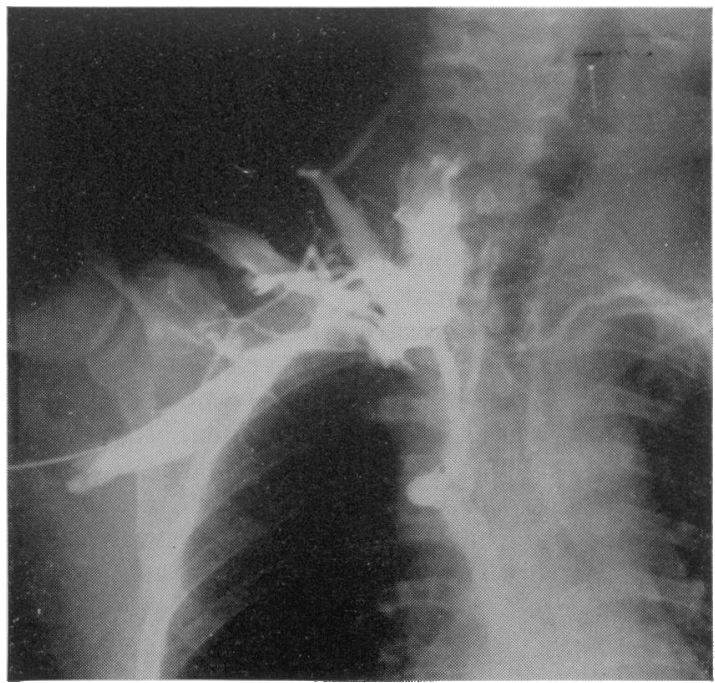

FIG. 5. Right subclavian venogram showing obstruction of the superior vena cava. 
few days following admission he developed an aphthous ulcer on the tongue. There was no clubbing, lymphadenopathy or peripheral phlebitis. Full ophthalmological examination revealed no evidence of uveitis. A straight $\mathrm{X}$-ray of the chest showed mediastinal widening and a right subclavian venogram was performed on 28 November (Dr R. G. Grainger) (Fig. 5).

The subclavian vein was dilated and completely occluded at its inner end. There was marked reflux into the right jugular vein and other neck veins, mainly on the right. A large vein conveyed contrast medium to the azygos vein in which it passed retrogradely downwards. Very little opacification of the right atrium was observed due to the very slow entry of contrast medium. The venous pressure in the right arm was extremely high.

Other investigations were as follows: $\mathrm{Hb}$ : $13.0 \mathrm{~g} / 100 \mathrm{ml}$. WBC: $13,600 / \mathrm{mm}^{3}$ (neutrophilia). ESR : $82 \mathrm{~mm} / \mathrm{hr}$ (Westergren). Throat swab: no pathogens on culture. Swab from scrotal ulcers: sterile. ASO titre: 100 Todd Units. Blood culture: sterile after 7 days incubation. WR, Kahn, Reiter: negative. LE cell preparation: no LE cells seen. Plasma proteins: albumin $3 \cdot 8$, globulin $2.9 \mathrm{~g} / 100 \mathrm{ml}$. Electrophoresis showed a slight increase in $\gamma$-globulin.

On 6 December a right thoracotomy was performed (Mr D. G. Taylor). The right lung was densely adherent to the mediastinum which was extremely vascular. Both the superior vena cava and the azygos vein were solid with thrombus. Due to technical difficulties an attempt to disobliterate the superior vena cava was not made, but the azygos vein was freed from clot and a way made for drainage through the azygos vein to the superior vena cava and right atrium. Biopsies were taken from the mediastinum and the wall of the superior vena cava. Bronchoscopy revealed no abnormality.

On histological examination the tissue from the pleura showed only dense fibrous tissue with hyalinization. Few cells were present and there was no evidence of active inflammation. The vena cava contained recent laminated thrombus with early organization at the periphery. None of the vein wall was present and no organisms could be demonstrated. The mediastinal material consisted of areolar tissue showing a mild nonspecific inflammation with areas of fibrinous exudate and formation of granulation tissue. Several small veins were occluded by recent thrombus but there was no evidence of arterial degeneration. No granulomata or giant cells were present (Fig. 6).

Initially, the patient had a stormy post- operative course during which he developed thrombosis of his left antecubital veins despite anticoagulant therapy. He gradually improved and his neck size returned to normal.

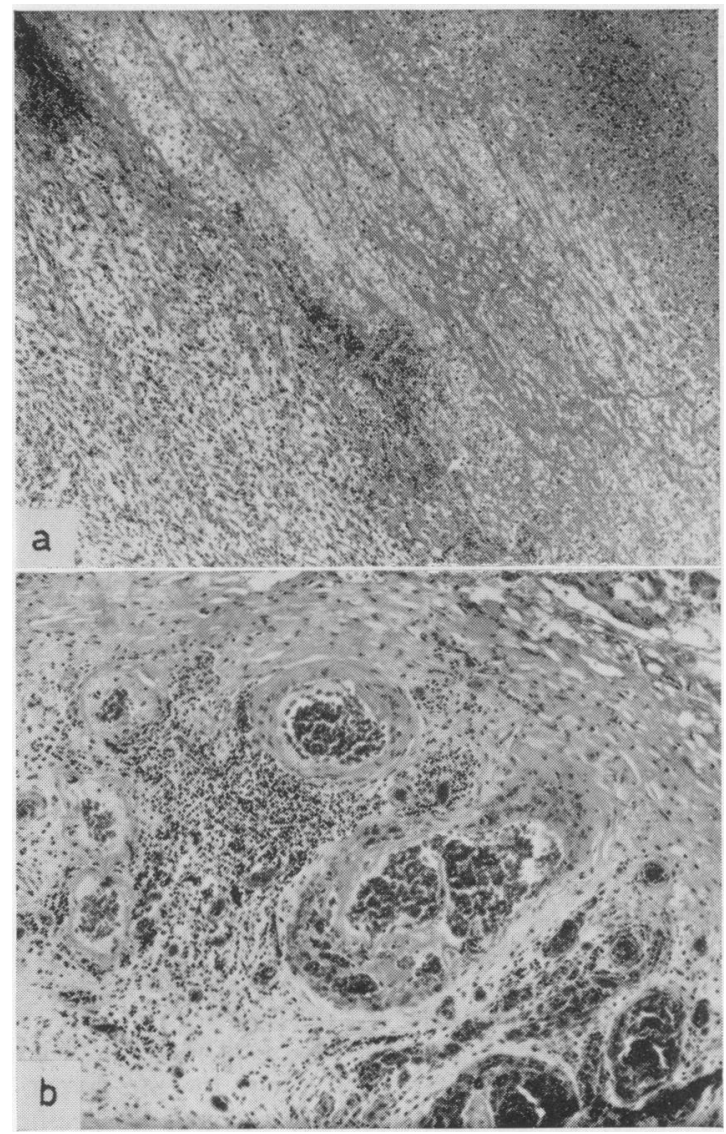

FIG. 6. (a) Organizing thrombus from the superior vena cava (H \& E, $\times 50$ ). (b) Mediastinal tissue showing vascular thrombosis and focal inflammation (H \& $E$, $\times 56)$.

A second right subclavian venogram was performed on 11 January 1967 (Dr R. G. Grainger).

The subclavian vein was considerably less dilated but there was still complete occlusion of its medial end. The opacification of the cervical veins was not as marked as on the earlier examination. The azygos vein again conveyed contrast medium retrogradely away from the right atrium. The right intercostal veins opacified from the axillary vein and carried contrast medium retrogradely towards the sternum. The pressure in the right arm veins was considerably less than on the earlier examination (Fig. 7).

When last seen, the patient remained well but 
still had complete block of his superior vena cava and persistent aphthous ulceration. Steroid therapy was not given at any stage.

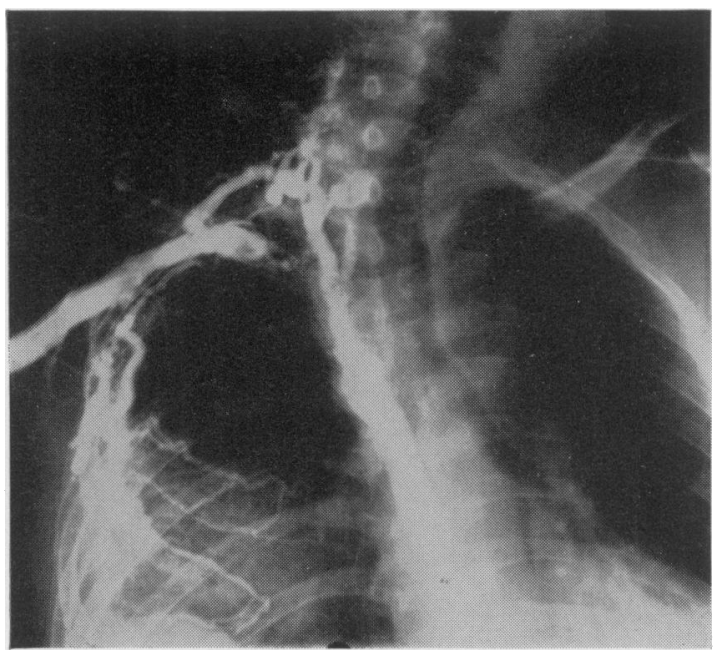

FIG. 7. Right subclavian venogram after operation.

\section{Discussion}

Theories advanced to explain the pathogenesis of Behçet's syndrome include those of rheumatic disease, bacterial allergy, viral infection and autoimmunity, and although there is a certain amount of evidence in support of each of these, none has been widely accepted or confirmed (Oshima et al., 1963). Current opinion favours the concept that the syndrome is basically a disease of blood vessels, probably a manifestation of an hyperergic state as shown by the hyperreactivity of the skin not only towards a specific antigen Behçetin, but following other stimuli of varied nature.

In the case described by Mishima et al. a 38-year-old male with Behçet's syndrome underwent a successful resection of an aortic aneurysm which was replaced by a graft. Histology of the aneurysm showed thickening of the adventitia, disruption of the elastic fibres of the media, hypertrophy of the intima and occlusion of the lumen by thrombus. Although Oshima et al. refer to two cases of Behçet's syndrome in which arterial aneurysms occurred, they describe only one. This was an aneurysm of the right ulnar artery in a woman aged 36 years. The aneurysm was resected and histology showed that 'small arteries near to aneurysm' had obstruction of their lumina with fibrous tissue, swelling of the lamina elastica interna, splitting of the elastic fibres of the media, and slight perivascular cell infiltration. In the case reported from Hammersmith Hospital a 24-year-old man with Behçet's syndrome died following rupture of an abdominal aortic aneurysm. At autopsy there was septic inflammation of the aortic wall and an old septic osteomyelitis of the adjacent vertebral bodies so that the aneurysm in this case may not have been due to a primary inflammatory lesion of the aorta. Our sections show some similar features to the previously reported cases but in addition emphasize that a primary acute arteritis may be a feature of Behçet's syndrome although the histological appearances are in no way specific. In the case reported by Hills, a male patient with Behçet's syndrome developed aneurysms of the thoracic and abdominal aorta, but histological studies of the lesions were not made.

The most frequent complication of popliteal aneurysms is acute thrombosis of the sac which suddenly occludes the lumen of the popliteal artery. In a recent study of twenty patients with twenty-nine popliteal aneurysms (Hara, 1967), acute obstruction developed in thirteen cases terminating in supra-condylar amputation of eight extremities. One required bilateral amputation. In this series ten patients had grafting with either autogenous saphenous vein or plastic 'Dacron' prosthesis with excellent results. In our first case we considered that severe pain and the danger of an acute episode of thrombosis were justifiable indications for surgery.

In the second case, a diagnosis of Behçet's syndrome was considered justified in spite of the absence of ocular lesions. Most authorities regard the diagnosis as tenable in the presence of two of the three features comprising the original triad (Mounsey, 1966).

Although no sections of the vena caval wall itself were obtained, the histological appearances of the adjacent tissues suggested that the vein wall had probably been involved in an inflammatory process. In this case operation was undertaken in the hope of carrying out some form of disobliterative or by-pass procedure. The former was not possible because of the extent of the thrombus, but by producing a free way to the right side of the heart through the azygos vein, we may have helped the patient during the acute stage. The vascular material affected by the disease was very poor and unsuitable for any sort of repair.

\section{Acknowledgments}

We wish to thank Professor C. H. Stuart-Harris and Dr E. G. Rhind for permission to publish these cases. We are also grateful to Mr Desmond Taylor, who performed all 
the operations, for his interest and encouragement, and to Miss Mary A. C. Jones for referring the first case. Histological material in the second case was kindly made available by $\operatorname{Dr}$ A. J. N. Warrack.

\section{References}

BEHÇET, H. (1937) Uber rezidivierende, aphthose, durch ein Virus Verursachte Geschune am Mund, am Auoe und an den Genitalien. Derm. Wschr. 105, 1152.

British Medical Journal (1965) Clinico-Pathological Conference. Postgraduate Medical School of London. Brit. med. J. i, 357.

Hılls, E.A. (1967) Behçet's syndrome with aortic aneurysms. Brit. med. J. iv, 152.
Forman, L. (1960) Thrombophlebitis und Arteritis in der Patholgie des Behçet-Syndroms. Hautarzt, 11, 363.

Forman, L. (1967) Vascular basis of the pathology of Behçet's disease. Excerpta Med. 16, Sect. 13.

Hara, M. (1967) The hazards of popliteal aneurysms. Surg. Gynec. Obstet. 124, 358.

Mishima, Y., Ishikawa, K. \& Kawase, S. (1961) Proc. Jap. Circ. Soc., Tokyo 1960. Jap. Circulat. J. 25, 12111.

MounSEY, J.P.D. (1966) Behçet's syndrome-a spectrum. Trans. St John's Hosp. derm. Soc. (Lond.), 52, 197.

Oshima, Y., Shimizu, Z., Yokohari, R., Matsumoto, T., Karino, K., KaOAmi, T. \& MaOaya, H. (1963) Clinical studies on Behçet's syndrome. Ann. rheum. Dis. 22, 36. 